\title{
Main factors affecting the genetic transformation of chrysanthemum var. Micromargara
}

\author{
Juan Ubaldo Sánchez-Velázquez ${ }^{1}$, Guadalupe López Puc ${ }^{1}$, Ana Luisa Ramos-Díaz ${ }^{1}$, Julia del Socorro \\ Cano-Sosa $^{1}$, Ingrid Mayanin Rodríguez Buenfil ${ }^{1}$, Rómulo García-Velasco ${ }^{2}$, y Alberto Uc Varguez ${ }^{{ }^{*}}$
}

\author{
${ }^{1}$ Centro de Investigación y Asistencia en Tecnología y Diseño del Estado de Jalisco AC., Unidad Sureste. Parque \\ científico y Tecnológico de Yucatán. Km. 5.5 Carretera Sierra Papacal-Chuburná Puerto. Cp. 97302, Mexico \\ ${ }^{2}$ Universidad Autónoma del Estado de México. Centro Universitario Tenancingo. Exhacienda de Santa Ana \\ Carretera Tenancingo-Villa Guerrero km 1.5 Tenancingo, México
}

\author{
*Corresponding author: auc@ ciatej.mx
}

\begin{abstract}
Factors influencing Agrobacterium tumefaciens- mediated genetic transformation of plants have been widely reported: type and concentration of antibiotic, co-culture period, concentration of bacteria, concentration of acetosyringone, and of course, the type and age of the explants, as well as temperature conditions. However, it is not yet understood how these factors interact and how they affect the efficiency of the final transformation. The aim of this work was to evaluate the interaction of the three main factors affecting the transformation of chrysanthemum (Dendranthema grandiflora) var. Micromargara. A $2^{3}$ factorial design was used with central points, in which three concentrations of A. tumefaciens $\left(1.0,1.5\right.$. and 2.0 of D.O. $\left.{ }_{600}\right)$, three concentrations of acetosyringone $(50 \mu \mathrm{M}, 75 \mu \mathrm{M}$ and $100 \mu \mathrm{M})$, and three co-culture periods (1, 2 and 3 days) were evaluated. The transformation was verified by GUS staining and by means of RT-PCR amplification and sequencing of fragment of the genes uidA, aph3 II and act-cr. The results show that, among the factors evaluated, only the concentration of A. tumefaciens presented a statistically significant effect $(p \leq 0.05)$ on transformation efficiency, without interaction with the factors of acetosyringone concentration and co-culture period. It is concluded therefore that an A. tumefaciens concentration of D.O ${ }_{600}: 2.0$ is determinant for greater transformation efficiency in chrysanthemum var. Micromargara.
\end{abstract}

Keywords: Chrysanthemum, Genetic transformation, Concentration of A. tumefaciens, co-culture period, concentration of acetosyringone.

Abbreviations: BAP_Benzylaminopurine, NAA_Naphthalene acetic Acid, MS_Murashige \& Skoog, PVP_Poly-(vinyl pyrrolidone), $N P T I I$ neomycin phosphotransferase II. GUS_ $\beta$ - glucoronidase.

\section{Introduction}

The chrysanthemum is one of the most important ornamental species in the world, with over 15000 cultivars reported (Teixeira da Silva and Kulus, 2014). Traditional improvement methods have been able to incorporate a few desirable characteristics into the species; however, due to the limitations resulting from the gene pool and crossincompatibilities, the traditional improvement is unable to satisfy the demands of the market. One strategy for developing new varieties and satisfying the demands of the consumer has been Agrobacterium-mediated genetic transformation (Naing et al., 2014; Opabode, 2006). This is a well-established procedure for a number of chrysanthemum varieties; however, one transformation protocol cannot be used in every variety of the same species (Shinoyama et al., 2012; Alimohammadi and Bagherieh-Najjar, 2009; Opabode, 2006) and that, in the case of the chrysanthemum, there are a number of recalcitrant varieties (Vences-Contreras et al., 2009). In order to carry out genetic transformation, plant tissue must be infected by means of co-culture with a variant of A. tumefaciens carrying a gene of interest. After coculture, the bacteria must be eliminated by transferring the plant tissue to a regeneration medium containing antibiotics in order to suppress bacterial growth; a procedure for the selection of transformed cells is also required (Teixeira da
Silva and Fukai, 2001). During the transformation, several components in the culture medium affect plant tissue, for example, the antibiotics used to suppress Agrobacterium can have a negative effect on tissue and regeneration (Farzaneh et al., 2013). With this in mind, the antibiotic no should it induce secondary effects in plant cells (Chung and Park, 2005; Teixeira da Silva and Fukai, 2001). Several reports have presented evidence of other factors affecting the genetic transformation of chrysanthemum, of which the most important are: the concentration of A. tumefaciens during infection, the use and concentration of inducers such as acetosyringone during infection and the co-culture (Takatsu et al., 2000; Ming et al., 2007), the extent of the co-culture period (Kudo et al., 2002; Teixeira da Silva and Fukai, 2003; Song et al., 2012). However, it is not clear how any one of these factors can affect others in a synergic process since the protocols focus on determining the effect of each factor individually and then using the best results from the best conditions evaluated in a single protocol, without taking into consideration the synergic effect or the adverse effect of one factor on the others which could affect transformation efficiency. The protocols developed for the genetic transformation of chrysanthemum are specific for the varieties used in said protocols and are very often inadequate 
or inefficient among the varieties of the same species. The aim of this work was to develop a method of $A$. tumefaciens mediated genetic transformation for chrysanthemum var. Micromargara and to evaluate the effect of three main factors and their interaction on genetic transformation.

\section{Results and Discussion}

\section{Main factors affecting genetic transformation of chrysanthemum}

Effect of inoculum concentration (1.0, 1.5 and 2.0 D.O ${ }_{600}$ of A. tumefaciens, measured by optic density at $600 \mathrm{~nm}$ ), acetosyringone concentration $(50,75$ and $100 \mu \mathrm{M})$ and coculture period (1,2 and 3 days) on the genetic transformation of calluses of chrysanthemum was tested using a $2^{3}$ factorial design (Table 1). GUS staining tests applied to the transformed calluses of chrysanthemum var. Micromargara were inconclusive in determining transformation efficiency due to the fact that no difference was observed between the fluorescence of transformed and non-transformed calluses. Thus, the results of GUS staining in the transformed calluses were discarded as evidence of transformation in this study. The lack of fluorescence in the transformed calluses may be due to the green color of the calluses used, as has been reported in other studies, in which an excess of chlorophyll and other pigments did not allow a clear differentiation between transformed and non-transformed explants. In addition, transformation efficiency was evaluated by RT-PCR assay directed to amplify a fragment of the genes uidA (GUS $690 \mathrm{bp}$ ) and aph'3 II (NPTII $490 \mathrm{bp}$ ) in transformed calluses. A fragment $670 \mathrm{bp}$ of the actin gene $(a c t-c r)$ was also amplified as a positive control for the expression (Figure 1). The identity of the fragments amplified (Figure 1) by RTPCR were confirmed by the analysis of the sequences obtained from purified and cloned fragments. Due to the fact that the sequences of all the genes amplified, uidA, aph3'II and act-cr, resulted in 100\% identity with sequences reported in the data base of NCBI, it is quite feasible to use the records of transformed calluses based on this evidence, as has been done in other reports (Kubo et al., 2006).

The analysis of the main effect (Figure 2) showed that only the factor of A. tumefaciens concentration presents a statistically significant effect in its highest value (Figure 3) for transformation efficiency. Moreover, an effect of the blocks was observed in the third repetition of the experiment (Table 1). This unexpected block effect could be explained by the age of the calluses used during the transformation; in blocks 1 and 2, calluses with an average of 4 weeks growth were used, while the calluses used in block 3 had an average of 1.5 weeks growth, with the latter showing a better performance regarding darkening, and a better appearance in general during the co-culture and disinfection phases of the transformation procedure. Arcos-Ortega et al (2010), observed a similar effect in leaf explants of habanero pepper (Capsicum chinense), while evaluating different ages of the explant at the moment of transformation, and reported that transformation efficiency was significantly reduced with the older explants, the argument being that these explants develop greater resistance to pathogens, including $A$. tumefaciens, thereby reducing the transformation events and allowing the development of necrotic tissue in the inoculation site.

The effect of A. tumefaciens concentration with an optic density of the inoculum of 2 was significant in experiments 1 , 5 and 6 with transformation efficiencies of $12.5 \%, 29.16 \%$ and $12.5 \%$, respectively (Table 1). Experiments 1 and 6 showed transformation efficiencies similar to the lowest transformation efficiencies obtained by Shinoyama et al (2002) for chrysanthemum var. Suho-no chicara, whereas the transformation efficiency obtained in experiment 7 was comparable to the highest efficiency reported by the same author (Shinoyama et al., 2002). The results of experiments 1 and 6 presented transformation efficiencies similar to those obtained by Song et al (2012), for the variety Orlando, in which a co-culture period of 2 days was evaluated, half the time period employed in this work, however, the results have shown that the co-culture period has no significant effect on the transformation efficiency of the variety Micromargara.

Other reports show lower transformation efficiencies (8.3\%), comparable to those obtained in experiment 3 , while the efficiencies obtained in experiments 1,5 and 6 were 2 to 5 times higher (Valizadeh et al., 2012). This may be because the co-culture periods of 1 and 2 days used in those experiments are insufficient to obtain more transformation events, while a co-culture period of 4 or more days induced Agrobacterium overgrowth (Kudo et al., 2002).

This work reports the efficient (29\%) transformation calluses of Chrysanthemum by A. tumefasciens. This could be extrapolated in principle to introduce any important gen in chrysanthemum. Given the development of new technologies that allow for gene editing, this transformation protocol stablished could be used for this end. For example CRISRPCas9 or RNAi have been used recently to introduce mutation in rice genes (Xu et al., 2015) and gen silencing for genetic improvement of several species (Koch and Kogel, 2014).

\section{Materials and Methods}

\section{Plant material and in vitro culture conditions}

Chrysanthemum plants (D. grandiflora) var. Micromargara with a lilac flower were propagated in vitro in MS medium (4.4 g/L of medium, containing 3\% sucrose and $0.2 \%$ Gelrite) supplemented with $1.0 \mathrm{mgL}^{-1}$ of Benzylaminopurine (BAP) and Polyvinyl polypyrrolidone (PVPP) at $0.1 \%$ (hereafter CC medium) using nodes with apical buds as initial explants. Leaf explants obtained from plants propagated in vitro were induced for callus formation in MS medium with $4.4 \mathrm{~g} / \mathrm{L}$ of medium containing $3 \%$ sucrose and $0.2 \%$ Gelrite, supplemented with $13.32 \mu \mathrm{M}$ BAP and $4.83 \mu \mathrm{M}$ of Naphthaleneacetic acid (NAA). Induction conditions were photoperiod of $16 \mathrm{~h}$ light, relative humidity $80 \%$ and a temperature of $25^{\circ} \mathrm{C} \pm 2^{\circ}$. Calluses $1.0 \mathrm{~cm}$ in size with 30 days of induction and replanted every 15 days were used in the genetic transformation.

\section{A. tumefaciens strain and transformation vector}

For the transformation of chrysanthemum calluses, the LBA4404 strain of A. tumefaciens containing the plasmid pAL4404 was used. This plasmid contains the vir region, the origin of replication and genes, which provide the antibiotics streptomycin and spectinomycin with resistance. As vector for the transformation, the binary plasmid pBI121 was used which contains, within the T-DNA region, the aph'3 II gene that codifies neomycin phosphotransferase II (NPTII) and the uidA gene which codifies $\beta$-glucuronidase (GUS). The introduction of the pBI121 vector into A. tumefaciens was performed by means of electroporation, according to previously described protocol (Wise et al., 2006). The Timentin antibiotic (Ticarcillin plus clavulanate potassium at a ratio of $10: 1)$ at $250 \mu \mathrm{g} / \mathrm{mL}$ of antibiotic added to the culture medium was used to select transformed calluses (In a previous test, it was determined that Timetin suppressed the 
Table 1. Percentage of transformed calluses positive to the RT-PCR evaluation for each treatment (coculture time, Agrobacterium concentration and Acetosiringone concentration) and repetition or block.

\begin{tabular}{lcccccc}
\hline Experiment & $\begin{array}{c}\text { Coculture time } \\
(\text { Days })\end{array}$ & $\begin{array}{c}\text { A.tumefaciens } \\
\text { concentration (DO) }\end{array}$ & $\begin{array}{c}\text { Acetosiringone } \\
\text { concentration }(\mu \mathrm{M})\end{array}$ & $\begin{array}{c}\text { *Block } \\
1\end{array}$ & Block 2 & Block 3 \\
\hline 1 & 3 & 2 & 100 & 0 & 0 & 37.5 \\
2 & 3 & 2 & 50 & 25 & 0 & 0 \\
3 & 3 & 1 & 100 & 0 & 0 & 25 \\
4 & 3 & 1 & 50 & 0 & 0 & 12.5 \\
5 & 1 & 2 & 100 & 0 & 37.5 & 50 \\
6 & 1 & 2 & 100 & 0 & 0 & 37.5 \\
7 & 1 & 1 & 50 & 0 & 0 & 50 \\
8 & 1 & 1 & & 0 & 0 \\
\hline
\end{tabular}

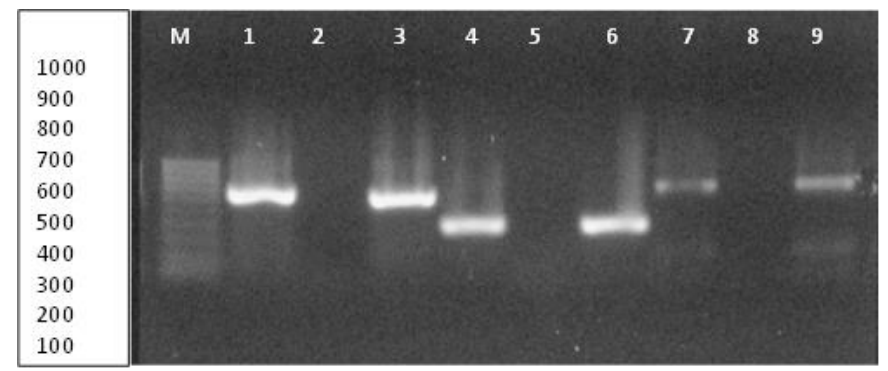

Fig 1. Electrophoresis in agarose gel of the products amplified by RT-PCR from uidA, aph3'II and act-cr. Molecular weight marker $1000 \mathrm{bp}(\mathrm{M})$, Positive control of uidA (1), Negative control of uidA (2), Amplification of a fragment of GUS from cDNA (3), Positive control of aph3'II (4), Negative control of aph3'II (5), 6) Amplified fragment of aph3'II from cDNA (6), 7) Positive control of act-cr (7), Negative control of act-cr (8), Fragment of act.cr amplified from cDNA (9).

Main Effects Plot for Transformantes

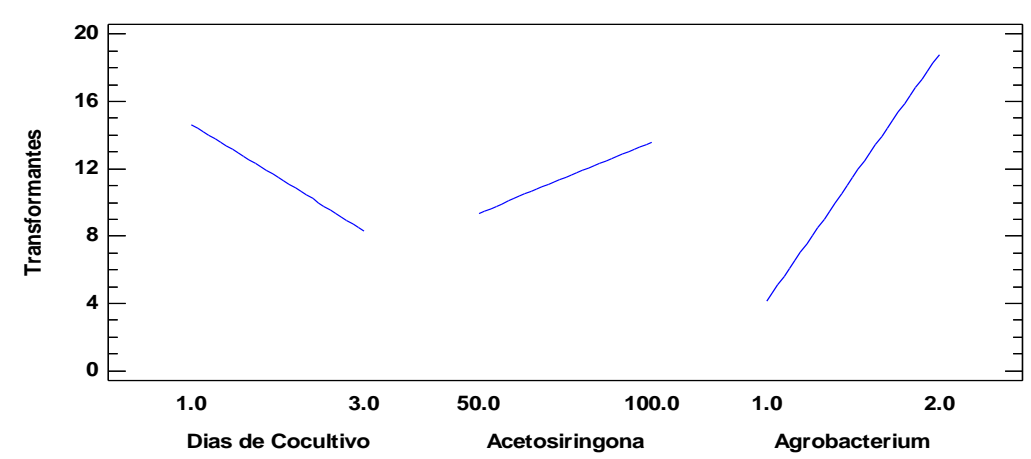

Fig 2. Main effects of the coculture time, acetosiringone and Agrobacterium concentration on callus transformation in chrysanthemum var. Micromargara (Degree of inclination of the bars indicates the impact on transformation). Interaction was found only between the co-culture period and acetosyringone concentration; however, the effect is not statistically significant.

Standardized Pareto Chart for Transformantes

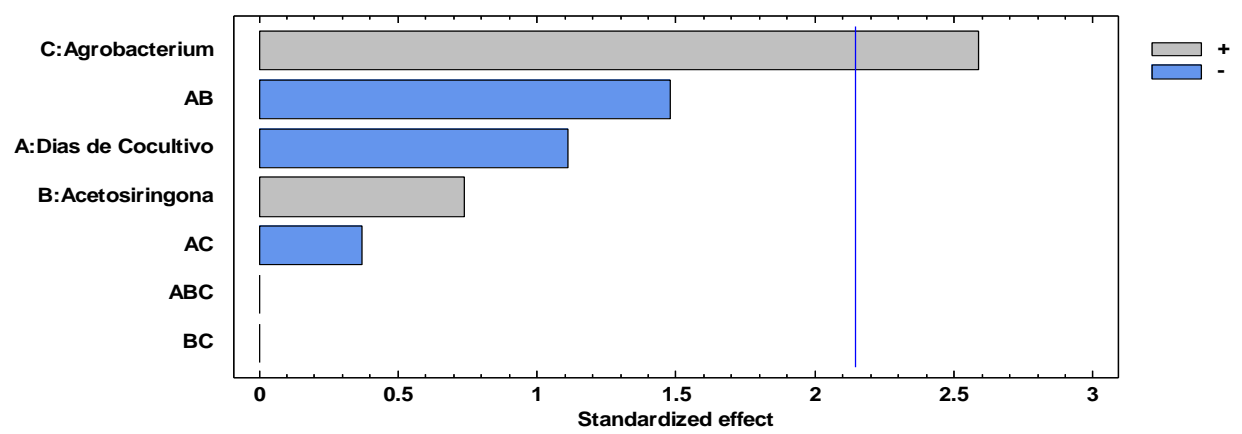

Fig 3. The analysis of statistical significant effect of each factor and effect of the interactions showed that only the factor of $A$. tumefaciens concentration presents a statistically significant effect $(p \leq 0.05)$ in its highest value. 
grown of $A$. tumefaciens and had no effect on the regeneration of transformed calluses) (No data presented).

\section{Main factors affecting genetic transformation of chrysanthemum}

In order to evaluate the effect of inoculum concentration (1.0, 1.5 and 2.0 D.O 600 of A. tumefaciens), acetosyringone concentration $(50,75$ and $100 \mu \mathrm{M})$ and co-culture period (1, 2 and 3 days) on the genetic transformation of chrysanthemum, a $2^{3}$ factorial design with central points was employed (Table 1). Eight calluses were used per treatment with three repetitions per treatment. The general process for the transformation of chrysanthemum calluses by $A$. tumefaciens, (Shinoyama et al., 2002), was performed by immersion in a transformation solution of $10 \mathrm{mM}$ glucose, acetosyringone for 20 minutes (Teixeira da Silva and Fukai, 2003), and variable concentrations of A. tumefaciens and acetosyringone depending on the treatment. The callus was placed in a co-culture medium (CC medium supplemented with the same concentration of acetosyringone used in the transformation solution) for periods of 1, 2 or 3 days. After co-culture, the callus was removed from the co-culture medium and rinsed twice with $250 \mathrm{mgL}^{-1}$ of Timentin and once more with sterile water; the callus was cultured in a disinfecting medium (CC medium, supplemented with 250 $\mathrm{mgL}^{-1}$ of Timentin) for 5 days, after which it was rinsed twice with $250 \mathrm{mgL}^{-1}$ of Timentin and once with sterile water. The callus was then cultured for a further five days in disinfecting medium (Shinoyama et al., 2002). At the end of this period, transformation efficiency was analyzed by means of the percentage of transformed calluses. A callus was considered transformed when tested positive to GUS staining and also fragments of the genes uidA and aphA3'II included in the transformation cassette were amplified by RT-PCR from RNA from the same callus.

\section{GUS staining}

The commercial kit " $\beta$-Glucuronidase Reporter Gene Staining Kit"was performed, following to the protocol recommended by the supplier (Sigma Aldrich), after which it was incubated at $37^{\circ} \mathrm{C}$ for $24 \mathrm{~h}$. The callus was then rinsed twice with ethanol at $70 \%$ and once with acetone at $50 \%$, according to the recommendations of Jefferson (1987). Finally, the number of explants presenting blue coloring and their percentage per treatment was registered by stereoscopic observation.

\section{Amplification of uidA, aphA3'II and act-cr by means of RT-PCR}

RNA extracted from $600 \mathrm{mg}$ of transformed callus, following the protocol of the trizol (Invitrogen), with some modifications. The RNA extracted was treated with the kit Turbo DNase free to eliminate DNA residue, according to the protocol recommended by the supplier (Ambion ${ }^{\circledR}$ ). RNA free of DNA was used to synthesize cDNA using the enzyme SuperScript ${ }^{\mathrm{TM}}$ II Reverse Transcriptase MMLV RT according to the protocol recommended by the supplier (Invitrogen).

Platinum Taq DNA Polymerase high fidelity and Specific primers were used: F1GUS (ctgtagaaaccccaaccegtg) and R1GUS (aaatccegctagtgcettgtccagttgc) to amplify a fragment of $690 \mathrm{bp}$ of the gen uidA; FNPTII (gagaggctattcggctatga) and RNPTII (gatgctcttcgtccagatca) to obtain a fragment of $490 \mathrm{bp}$ of the gen aph3'II and finally, the primers FACT1 (tggagaaaatctggcatcacac) and RACT1 (ggaacctagttgtaccaccac) were used to amplify a fragment of $670 \mathrm{bp}$ of the gen act-cr (Teixeira da Silva and Fukai, 2003). Conditions of amplification of the gene uidA was performed by means of Touch-down, beginning with a cycle of $94^{\circ} \mathrm{C} / 1$ min, followed by $65^{\circ} \mathrm{C} / 2 \mathrm{~min}$ and finally, $72^{\circ} \mathrm{C} / 2 \mathrm{~min}$; the subsequent cycles dropped $1^{\circ} \mathrm{C}$ during alignment of the primers, to $58^{\circ} \mathrm{C}$, after which 35 cycles of $94^{\circ} \mathrm{C} / 1 \mathrm{~min}$, $58^{\circ} \mathrm{C} / 2 \mathrm{~min}, 72^{\circ} \mathrm{C} / 2 \mathrm{~min}$ and a final extension of $72^{\circ} \mathrm{C} / 10$ min were performed. The amplification of aph $3^{\prime} I I$ was performed with touchdown, beginning with a cycle of $94^{\circ} \mathrm{C} / 1$ min, followed by $58^{\circ} \mathrm{C} / 2 \mathrm{~min}$ and finally, $72{ }^{\circ} \mathrm{C} / 2 \mathrm{~min}$, the subsequent cycles dropped $1{ }^{\circ} \mathrm{C}$ during alignment of the primers, to $50^{\circ} \mathrm{C}$, after which 35 cycles of $94^{\circ} \mathrm{C} / 1 \mathrm{~min}$, $54^{\circ} \mathrm{C} / 2 \mathrm{~min}$ and a final extension of $72^{\circ} \mathrm{C} / 10 \mathrm{~min}$. were performed. For the amplification of act-cr, the thermal program used was one cycle of $95^{\circ} \mathrm{C} / 3 \mathrm{~min}$, followed by 25 cycles of $94^{\circ} \mathrm{C} / 1 \mathrm{~min}, 60^{\circ} \mathrm{C} / 2 \mathrm{~min}, 72^{\circ} \mathrm{C} / 2 \mathrm{~min}$ and a final extension of $72^{\circ} \mathrm{C} / 10 \mathrm{~min}$. The amplified fragments were analyzed by electrophoresis in $1 \%$ agarose gel, after which the gel was visualized in a photo documenter (UVP). The transformed calluses in which a gene fragment was amplified by RT-PCR were considered positive. The identity of the amplified fragments was confirmed by sequencing, in which the amplified bands were excised from the agarose gel and purified using the purelink quick plasmid miniprep kit (Invitrogen). The purified fragments were cloned and sent to Macrogen Korea for sequencing using standard protocols. The resulting sequences were edited and analyzed using the edit sequence program (Lassergene package DNASTAR ver 5.08 Madison USA), after which they were compared to the sequences reported in the data base of the NCBI GenBank using the BLAST program, available on the Internet.

\section{Statistical analysis of the transformed calluses of chrysanthemum}

The results of callus transformation in chrysanthemum obtained in the $2^{3}$ factorial experimental design was subjected to an analysis of main effects including ANOVA. To showing the significance of each factor, as well as the significance of the combination of factor at both high and low levels a Pareto analysis was performed with a maximum of three interactions. Finally, an analysis of interaction was performed with statistically significant factors, with aid of the statistical program Statgraphics centurion XVI.0.

\section{Conclusions}

We found that the most effective conditions for genetic transformation of Chrysantemum (D. grandiflora) var Micromargara, mediated by A. tumefaciens was: bacteria concentration of OD ${ }_{600}: 2$, with one day of co culture time and $100 \mu \mathrm{M}$ of acetosyringone concentration. The concentration of A. tumefaciens presented a statistically significant effect on transformation efficiency ( $p \leq 0.05)$, without interaction with the factors of acetosyringone concentration and co-culture period. Furthermore, an $A$. tumefaciens concentration of D.O ${ }_{600}: 2.0$ is determinant for greater transformation efficiency in chrysanthemum var. Micromargara.

\section{Acknowledgements}

This work was financed with resources from the SEPCONACyT Basic science Project, "Genetic modification of the biosynthesis route of anthocianines in chrysanthemum" code number, 2856 


\section{References}

Alimohammadi M and Bagherieh-Najjar M (2009) Agrobacterium- mediated transformation of plants: basic principles and influencing factors. Afr J Biotechnol. 8: 5142-5148.

Arcos-Ortega GF, Chan-Kuuk RA, González-Kantún WA, Souza-Perera R, Nakazawa-Ueji YE, Avilés-Berzunza E, Godoy-Hernández G, Lawton MA and Zúñiga-Aguilar JJ (2010) Agrobacterium tumefaciens-transient genetic transformation of habanero pepper (Capsicum chinense Jacq.) leaf explants. Electron J Biotechn. 13:1-9.

Chung K-M and Park Y-D (2005) Development of an agrobacterium-mediated transformation system for regeneration garland chrysanthemum (Chrysanthemum coronarium L.). J Plant Biol. 48: 136-141.

Farzaneh A, Adel Y, Ali N and Younes G (2013) Determine effective concentrations of B-lactam antibiotics against three strains of Agrobacterium tumefaciens and phytotoxicity on tomato and tabacco. Intl J Agron Plant Prod. 4: 2919-2925.

Jefferson RA (1987) Assaying chimeric genes in plants: the GUS gene fusion system. Plant Mol Report. 5: 387-405.

Koch A, and Kogel K H (2014) New wind in the sails: improving the agronomic value of crop plants through RNAi-mediated gene silencing. Plant Biotechnol J. 12: 821-831

Kubo T, Tsuro M, Tsukimori A, Shizukawa Y, Takemoto T, Inaba K and Shiozaki S (2006) Morphological and physiological changes in transgenic Chrysanthemum morifolium Ramat. ogura-nishiki with rol C. J Jap Soc Hort Sci. 75: 312-317.

Kudo S, Shibata N, Kanno Y, Suzuki M (2002) Transformation of chrysanthemum (Dendranthema grandiflorum (Ramat.) Kitamura) via Agrobacterium tumefaciens. Acta Hort. 572: 139-143.

Ming O, Wen A, Sinniah U and Subramaniam S (2007) Cysteine and acetosyringone are the two important parameters in agrobacterium-mediated transformation of rose hybrid (Rosa hybrida L.) cv. Nikita. J Plant Sci. 2: 387-397.

Naing A H, Park K I, Lim S H and Kim C K (2014) Appropriate choice of antibiotics for plant regeneration and optimization of selective agents to be used in genetic transformation for chrysanthemum. Plant Omics. 7: 237243.

Opabode J T (2006) Agrobacterium mediated transformation of plants: emerging factors that influence efficiency. Biotechnol Mol Biol Rev. 1: 12-20.

Shinoyama H, Aida R, Ichikawa H, Nomura $\mathrm{Y}$ and Mochizuki A (2012) Genetic engineering of chrysanthemum (Chrysanthemum morifolium): current progress and perspectives. Plant Biotechnol. 29: 323-337.
Shinoyama H, Kazuma T, Komano M, Nomura Y and Tsuchiya T (2002) An efficient transformation system in chrysanthemum (Dendranthema grandiflorum (Ramat.) Kitamura) for stable and non-chimeric expression of foreign genes. Plant Biotechnol. 19: 335-343.

Song J, Sivanesan I and Jeong B (2012) Use of petal explants for successful transformation of Dendranthema grandiflorum Kitamura "Orlando" mediated by Agrobacterium tumefaciens. Afr J Biotechnol.11: 91419148.

Takatsu Y, Hayashi M and Sakuma F (2000) Transgene inactivation in agrobacterium-mediated chrysanthemum (Dendranthema grandiflorum (Ramat.) Kitamura) transformants. Plant Biotechnol. 17: 241-245.

Teixeira da Silva JA and Fukai S (2003) Four gene introduction methods affect the shoots regeneration and localization of transgene expression in greenhouse stem explants and in vitro-grown chrysanthemum stem thin cell layers. Afr J Biotechnol. 2: 114-123.

Teixeira da Silva JA and Fukai S (2001) The impact of carbenicillin, cefotaxime and vancomycin on chrysanthemum and tobacco TCL morphogenesis and agrobacterium growth. J Appl Hort. 3: 3-12.

Teixeira da Silva JA and Kulus D (2014) Chrysanthemum biotechnology: discoveries from the recent literature. Folia Hort. 26: 67-77.

Valizadeh M, Kazemitabar S and Jongsma M (2012) Agrobacterium mediated genetic transformation of chrysanthemum (Chrysanthemum morifolium Ramat.) with an aphidical gene, gcs (gamma-cadinene sintase). Int $\mathrm{J}$ Plant Breed Genet. 6: 1-14.

Vences-Contreras C, Vazquez-García LM and HernandezRodriguez OA (2009) Regeneración in vitro de once cultivares de crisantemo (Dendranthema grandiflora Tzvelev) a partir de meristemos apicales. Agron Mesoam. 20: 409-415.

Wise A, Liu Z and Binns A (2006) Three methods for introduction of foreigns DNA into Agrobacterium. In: Wang, K (ed) Agrobacterium protocols, 2da edn. Humana Press Inc., New York USA.

Xu R-F, Li H, Qin R-Y, Li J, Qiu C-H, Yang Y-C, Ma H, Li L, Wei P-C and Yang J-B (2015) Generation of inheritable and "transgene clean" targeted genome-modified rice in later generations using the CRISPR/Cas9 system. Sci Rep. 5: 11491 . 